\title{
Stability, Trajectory Following and Formation Control of Wheel Mobile Robot
}

\author{
Muhammad Farhan Manzoor ${ }^{1, *}$, Qinghe $\mathrm{Wu}^{1}$, and Rana Javed Masood ${ }^{2}$ \\ ${ }^{I}$ School of Automation, Beijing Institute of Technology Beijing, Beijing 100081, China \\ ${ }^{2}$ College of Automation, Nanjing University of Aeronautics and Astronautics Nanjing, Nanjing 210016, China
}

\begin{abstract}
This paper comprises of two parts, the first one is concerned with controlling a wheeled mobile robot, where the robot is trained to follow a trajectory and the second part is an extension of controlling of the robots by following a trajectory while maintaining their formation intact. Unicycle kinematics is considered for the control design of each robot, and the leader-follower structure for the formation. It is assumed that every robot except the one located at the end of each team, can potentially be a leader to the one behind it. It is also assumed that each follower is capable of sensing its relative distance and relative velocity with respect to its preceding robot. The stability of the control law is also proposed, that is investigated in the case of perfect sensing and in the presence of input saturation. The impact of measurement noise on the followers is then studied assuming that a known upper bound exists on the measurement error, and a linear matrix inequality (LMI) methodology is proposed to design a control law which minimizes the upper bound on the steady-state error. Matlab Simulations are presented to demonstrate the efficacy of the results obtained in this paper.
\end{abstract}

Keywords: Measurement noise, linear matrix inequality, formation, Matlab.

\section{INTRODUCTION}

Robotics has been of interest to mankind for over one hundred years. Robotics brings revolution in the field of engineering, that has established its effectiveness in industrial manufacturing, design, construction and operation. The advantages of using robots instead of humans for performing some specific tasks (e.g., underwater exploration, military surveillance, Search and Rescue operations, agriculture cultivation of land and handling hazardous material) have attracted many researchers. This research is mainly focused on the mobile robots.

This paper first considers the design of control law for the nonholonomic dynamics of the unicycle robot, and then extends it to the team of robots for formation. Considering the nonholonomic dynamics of the system, it is difficult finding control input for posture stabilization of the system. It is often desirable to design control laws under which the system variables converge to a trajectory, rather than to a point. In this type of problem, the desired trajectory is predefined and the robot is to be controlled so that it asymptotically converges to the desired path. Various trajectory tracking methods are given in the literature. This includes using nonlinear feedback laws, dynamic feedback linearization, and backstepping approach, to name only a few. To design the control inputs to guarantee the stability of the closed-loop system, it is assumed that there is "perfect velocity tracking." Reference [1] proposed an error based tracking model and

*Address correspondence to this author at School of Automation, Beijing Institute of Technology Beijing, Beijing 100081, China;

Tel: +8613681244542; E-mail: farhan_manzoor@hotmail.com designed a stable kinematic tracking controller for the nonholonomic mobile robot. Reference [2] studied the tracking control problem for nonholonomic mobile robots with limited information of a desired trajectory. The control law for trajectory tracking presented $\lceil 3\rceil$ is derived from a method developed for unicycles and is based on a real time combination of static linear feedbacks that are obtained by an off line LMI (Linear Matrix Inequalities) approach. Extensive publication on trajectory tracking of nonholonomic systems such as mobile robots can be found in the literature (see e.g., [4-9]).

Coordination of multiple mobile robots, on the other hand, has attracted much interest recently. Exploiting a group of robots instead of a single robot or human for performing a prescribed spatially distributed task has significant advantages in various applications [10].

Formation control is one of the most challenging research problems in cooperative mobile robots. This problem is concerned with a group of robots moving in formation and performing a single mission in a cooperative fashion. It is desired in this type of problem to control the relative position and orientation of the robots with respect to each other.

Reference [11] presents a kinematic controller based on the receding horizon leader-follower (RH-LF) control framework to solve the formation problem of multiple nonholonomic mobile robots. Reference [12] proposed a kinematic controller for the distributed consensus-based formation control. However, the perfect velocity tracking assumption does not hold in practice, and the dynamics of robot should not be ignored and practical control strategies accounting for both the kinematic and dynamic affect should be implemented [13-15]. 

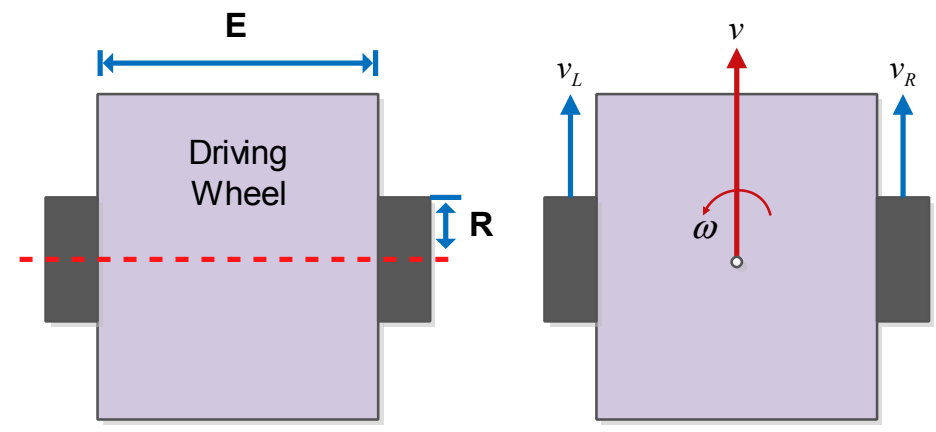

Fig. (1). Top view of wheeled type mobile robot.

Applications of formation control of cooperative robots include simultaneous localization and mapping, RoboCup (which is designed to play soccer and perform search and rescue), exploration of an unknown environment, and transportation of large objects, to name only a few [16-19].

\section{MOBILE ROBOT KINEMATICS}

The mechanical structure of a simple Wheeled Type mobile robot is shown in Fig. (1). The wheeled mobile robot is a classic non-holonomic system with two driving wheels controlled autonomously [20].

If the angular velocity of the left and right wheels is defined as $\omega_{L}, \omega_{R}$, respectively, the linear velocity of the left and right wheel $\mathrm{v}_{\mathrm{L}}, \mathrm{v}_{\mathrm{R}}$ is computed using

$\mathrm{v}_{\mathrm{R}}=\omega_{\mathrm{R}} \mathrm{R}, \mathrm{v}_{\mathrm{L}}=\omega_{\mathrm{L}} \mathrm{R}$

Where $\mathrm{R}$ is the radius of the wheel. Using the velocities of the two driving wheels $\mathrm{v}_{\mathrm{L}}, \mathrm{v}_{R}$, the linear and angular velocities of the robot are computed using (2)

$\mathrm{v}=\frac{\mathrm{v}_{\mathrm{R}}+\mathrm{v}_{\mathrm{L}}}{2}, \omega=\frac{\mathrm{v}_{\mathrm{R}}-\mathrm{v}_{\mathrm{L}}}{\mathrm{E}}$

where $\mathrm{E}$ is the length of the wheel base of the mobile robot.

\subsection{Problem Formulation}

Let $\mathrm{z}=\mathfrak{R}^{\mathrm{n}}$ denote the set of all $n$-vectors of generalized coordinates for a wheeled mobile robot.

The generalized coordinates for a unicycle are $\mathrm{z}=(\mathrm{x}, \mathrm{y}, \theta)$, where $(\mathrm{x}, \mathrm{y})$ represents the Cartesian coordinates and $\theta$ is the angular orientation with respect to the $\mathrm{x}$-axis in an inertial reference frame. The objective here is to control the robot in such a way that it follows a certain trajectory with a desired velocity. Let the inertial reference frame be centered at the origin $O$ of the plane (Fig. (2). The differential equations describing the motion of the robot with respect to this frame are:

$$
\begin{gathered}
\dot{\mathrm{x}}=\mathrm{v} \cos \theta \quad \dot{\mathrm{y}}=\mathrm{v} \sin \theta \\
\theta=\omega \quad \dot{\mathrm{v}}=\mathrm{a}
\end{gathered}
$$

Where the acceleration a is treated as the input variable.

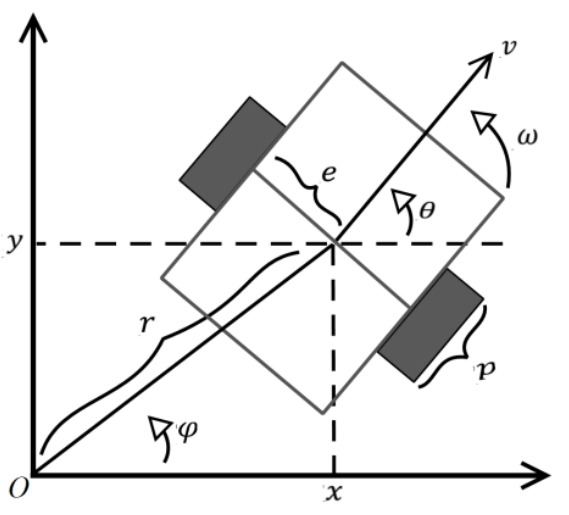

Fig. (2). Unicycle model.

The error vector is subsequently defined as:

$\mathrm{e}=\left[\begin{array}{c}\mathrm{e}_{\mathrm{p}} \\ \mathrm{e}_{\mathrm{v}}\end{array}\right]$

where $e_{p}$ and $e_{v}$ are the position and velocity error of the robot, respectively, and are defined by:

$\mathrm{e}_{\mathrm{p}}:=\left[\begin{array}{c}\mathrm{e}_{\mathrm{p}_{\mathrm{x}}} \\ \mathrm{e}_{\mathrm{p}_{\mathrm{y}}}\end{array}\right]=\left[\begin{array}{c}\mathrm{x}-\mathrm{x}_{\mathrm{r}} \\ \mathrm{y}-\mathrm{y}_{\mathrm{r}}\end{array}\right]$

$\mathrm{e}_{\mathrm{v}}:=\left[\begin{array}{l}\mathrm{e}_{\mathrm{v}_{\mathrm{x}}} \\ \mathrm{e}_{\mathrm{v}_{\mathrm{y}}}\end{array}\right]=\left[\begin{array}{l}\mathrm{v} \cos \theta-\mathrm{v}_{\mathrm{x}_{\mathrm{r}}} \\ \mathrm{v} \sin \theta-\mathrm{v}_{\mathrm{y}_{\mathrm{r}}}\end{array}\right]$

where $\left(\mathrm{x}_{\mathrm{r}}, \mathrm{y}_{\mathrm{r}}\right)$ is the reference value (set point) for the position of the robot, and $\left(\mathrm{v}_{\mathrm{x}_{\mathrm{r}}}, \mathrm{v}_{\mathrm{y}_{\mathrm{r}}}\right)$ is the reference value for the velocity of the robot. It is assumed that the robot is equipped with the proper sensors to measure its relative position and velocity (with respect to the desired set points).

Thus, the error vector $e$ can be used in constructing the control input. Now, using equations (5) and (6) one can find:

$\dot{\mathrm{e}}_{\mathrm{p}}=\left[\begin{array}{c}\dot{\mathrm{e}}_{\mathrm{p}_{\mathrm{x}}} \\ \dot{\mathrm{e}}_{\mathrm{p}_{\mathrm{y}}}\end{array}\right]=\left[\begin{array}{l}\mathrm{v} \cos \theta-\mathrm{v}_{\mathrm{x}_{\mathrm{r}}} \\ \mathrm{v} \sin \theta-\mathrm{v}_{\mathrm{y}_{\mathrm{r}}}\end{array}\right]$ 
and similarly

$\dot{\mathrm{e}}_{\mathrm{v}}=\left[\begin{array}{c}\dot{\mathrm{e}}_{\mathrm{v}_{\mathrm{x}}} \\ \dot{\mathrm{e}}_{\mathrm{v}_{\mathrm{y}}}\end{array}\right]=\left[\begin{array}{c}\dot{\mathrm{v}} \cos \theta-\mathrm{v} \dot{\theta} \sin \theta-\mathrm{a}_{\mathrm{x}_{\mathrm{r}}} \\ \dot{\mathrm{v}} \sin \theta+\mathrm{v} \dot{\theta} \cos \mathrm{q}-\mathrm{a}_{\mathrm{y}_{\mathrm{r}}}\end{array}\right]$

where

$a_{x_{r}}=\frac{d}{d t}\left[v_{x_{r}}(t)\right]$

and

$\mathrm{a}_{\mathrm{y}_{\mathrm{r}}}=\frac{\mathrm{d}}{\mathrm{dt}}\left[\mathrm{v}_{\mathrm{y}_{\mathrm{r}}}(\mathrm{t})\right]$

By rewriting the above equations and using the relations (3), it can be shown that:

$\dot{\mathrm{e}}_{\mathrm{v}}=\left[\begin{array}{c}\dot{\mathrm{e}}_{\mathrm{v}_{\mathrm{x}}} \\ \dot{\mathrm{e}}_{\mathrm{v}_{\mathrm{y}}}\end{array}\right]=\left[\begin{array}{cc}\cos q & -\mathrm{v} \sin q \\ \operatorname{sinq} & \mathrm{v} \cos q\end{array}\right]\left[\begin{array}{c}\mathrm{a} \\ \mathrm{w}\end{array}\right]=\left[\begin{array}{c}\mathrm{a}_{\mathrm{x}_{\mathrm{r}}} \\ \mathrm{a}_{\mathrm{y}_{\mathrm{r}}}\end{array}\right]$

Define

$\left[\begin{array}{l}u_{1} \\ u_{2}\end{array}\right]=\left[\begin{array}{rr}\cos \theta & -v \sin \theta \\ \sin \theta & v \cos \theta\end{array}\right]\left[\begin{array}{c}\mathrm{a} \\ \mathrm{w}\end{array}\right]$

Hence, (7) can be expressed as:

$\left[\begin{array}{c}\dot{\mathrm{e}}_{\mathrm{v}_{\mathrm{x}}} \\ \dot{\mathrm{e}}_{\mathrm{v}_{\mathrm{y}}}\end{array}\right]=\left[\begin{array}{c}\mathrm{u}_{1} \\ \mathrm{u}_{2}\end{array}\right]-\left[\begin{array}{c}\mathrm{a}_{\mathrm{x}_{\mathrm{r}}} \\ \mathrm{a}_{\mathrm{y}_{\mathrm{r}}}\end{array}\right]$

Combining the differential equations for the position and velocity errors, the state space representation of the system can be written as:

$\left[\begin{array}{c}\dot{\mathrm{e}}_{\mathrm{p}_{\mathrm{x}}} \\ \dot{\mathrm{e}}_{\mathrm{p}_{\mathrm{y}}} \\ \dot{\mathrm{e}}_{\mathrm{v}_{\mathrm{x}}} \\ \dot{\mathrm{e}}_{\mathrm{v}_{\mathrm{y}}}\end{array}\right]=\underbrace{\left[\begin{array}{cccc}0 & 0 & 1 & 0 \\ 0 & 0 & 0 & 1 \\ 0 & 0 & 0 & 0 \\ 0 & 0 & 0 & 0\end{array}\right]}_{A}\left[\begin{array}{c}\mathrm{e}_{\mathrm{p}_{\mathrm{x}}} \\ \mathrm{e}_{\mathrm{p}_{\mathrm{y}}} \\ \mathrm{e}_{\mathrm{v}_{\mathrm{x}}} \\ \mathrm{e}_{\mathrm{v}_{\mathrm{y}}}\end{array}\right]+$

$\underbrace{\left[\begin{array}{ll}0 & 0 \\ 0 & 0 \\ 1 & 0 \\ 0 & 1\end{array}\right]}_{B}\left[\begin{array}{c}u_{1} \\ u_{2}\end{array}\right]-\underbrace{\left[\begin{array}{ll}0 & 0 \\ 0 & 0 \\ 1 & 0 \\ 0 & 1\end{array}\right]}_{B}\left[\begin{array}{c}\mathrm{a}_{\mathrm{x}_{\mathrm{r}}} \\ \mathrm{a}_{\mathrm{y}_{\mathrm{r}}}\end{array}\right]$

or be equivalent to

$\dot{\mathrm{e}}=\mathrm{Ae}+\mathrm{Bu}-\mathrm{Ba}_{\mathrm{r}}$

where $a_{r}$ is the reference value for the acceleration of the robot in the $\mathrm{x}$ and $\mathrm{y}$ directions, and is expressed as: $a_{r}:=\left[\begin{array}{c}a_{x_{r}} \\ a_{y_{r}}\end{array}\right]$ form:

It is desired to design a control law of the following

$\mathrm{u}=\mathrm{Ke}+\mathrm{a}_{\mathrm{r}}:=\mathrm{a}+\mathrm{a}_{\mathrm{r}}$

to regulate the error defined by (4), where $K \in R^{2 \times 4}$ is a constant matrix.

\subsection{Main Results}

\subsubsection{Perfect Sensing without Input Constraint}

In this section, the stability of system (8) under the controller of the form (6), assuming that no error exists in sensor measurements is investigated.

Theorem 1. Consider a mobile robot following a desired trajectory, where the error dynamics of the robot is governed by (8). Given $\alpha>0$, suppose that there exist $R>0$ and $S$ satisfying the following LMI:

$\mathrm{RA}^{\mathrm{T}}+\mathrm{AR}+\mathrm{S}^{\mathrm{T}} \mathrm{B}^{\mathrm{T}}+\mathrm{BS}+\alpha \mathrm{R}<0$

Apply the controller (9) with $\mathrm{K}=\mathrm{SR}^{-1}$ to the robot; then $\|\mathrm{e}(\mathrm{t})\|$ is exponentially decaying (where $\|\cdot\|$ denotes the 2 norm).

\subsubsection{Perfect Sensing with Input Constraint}

Theorem 2. Consider the system described in Theorem 1 and let

$\mu_{1}=\max \left\|\mathrm{e}_{\mathrm{p}_{\mathrm{x}}}\right\|=\max \left\|\mathrm{e}_{\mathrm{p}_{\mathrm{y}}}\right\|$

and

$\mu_{2}=\max \left\|\mathrm{e}_{\mathrm{v}_{\mathrm{x}}}\right\|=\max \left\|\mathrm{e}_{\mathrm{v}_{\mathrm{y}}}\right\|$.

Given the design parameters $\alpha>0, \eta>0$, solve the following LMIs

$\mathrm{RA}^{\mathrm{T}}+\mathrm{AR}+\mathrm{S}^{\mathrm{T}} \mathrm{B}^{\mathrm{T}}+\mathrm{BS}+\alpha \mathrm{R}<0$

$\left[\begin{array}{cc}\eta^{2} R & S^{T} \\ S & I_{2}\end{array}\right]>0$
$R>4\left[\begin{array}{cc}\mu_{1}^{2} I_{2} & 0 \\ 0 & \mu_{2}^{2} I_{2}\end{array}\right]>0$

where $I_{2}$ is the $2 * 2$ identity matrix. Assume the problem has a feasible solution; then:

(i) If the controller (9) with $\mathrm{K}=\mathrm{SR}^{-1}$ is applied to the robot, then $\|e(t)\|$ is exponentially decaying, and 
(ii) ||$\mu||<\eta$

\subsubsection{Noisy Measurements}

In order to take into account the effect of measurement noise on the robot's motion, the control law (9) is modified as:

$$
u=K \tilde{e}+a_{r}
$$

where $\tilde{\mathrm{e}}=\mathrm{e}+\boldsymbol{\delta}_{\mathrm{e}}$, and $\boldsymbol{\delta}_{\mathrm{e}}$ is the measurement noise which is assumed to have a known bound represented by:

$$
\Delta_{\mathrm{e}}:=\underbrace{\max }_{\mathrm{t}>\mathrm{t}_{0}}\left\|\delta_{\mathrm{e}}\right\|^{2}
$$

In this subsection, an upper bound on the steady-state error is obtained and an algorithm is proposed to design $\mathrm{K}$ such that this upper bound is minimized.

Lemma 1. Given a positive scalar $\boldsymbol{\alpha}$ let the following inequality hold

$$
\dot{\mathrm{V}}+\alpha \mathrm{V}-\mathrm{b} \delta_{\mathrm{e}}^{\mathrm{T}} \mathrm{Q} \delta_{\mathrm{e}}<0
$$

where $\mathrm{b}$ is a positive constant and $\mathrm{Q}$ is a symmetric positive definite matrix. Then:

$$
\mathrm{V}(\infty)<\frac{\mathrm{b}}{\alpha} \underbrace{\max }_{\mathrm{t}>\mathrm{t}_{0}}\left[\delta_{\mathrm{e}}^{\mathrm{T}}(\mathrm{t}) \mathrm{Q} \delta_{\mathrm{e}}(\mathrm{t})\right]
$$

\section{EXTENSION OF PROPOSED METHOD TO FORMATION CONTROL WITH LEADER FOL- LOWER CONFIGURATION}

In this section, the problem of controlling a group of mobile robots following a trajectory while maintaining their formation intact is considered. The control design is carried out for the case of unicycle kinematics, which is the most common among wheeled mobile robots (WMR). It is assumed that every robot except the leader and the one located at the end of each platoon may potentially be a follower with respect to the one immediately in front of it, or a leader with respect to the one behind it (Fig. (3). The desired relative position of each follower with respect to its corresponding leader is assumed to be known by that follower. It is assumed also that each follower is capable of sensing its relative distance and relative velocity with respect to its preceding robot.

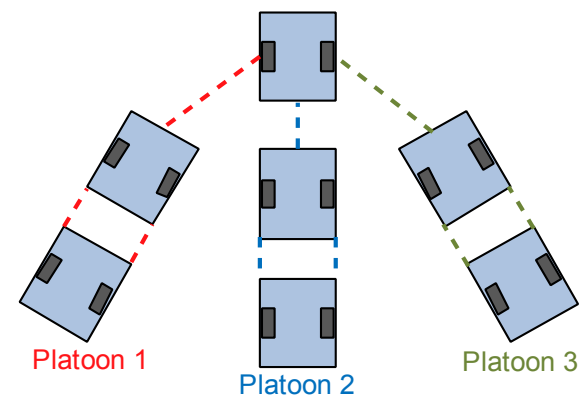

Fig. (3). Platoon of mobile robots.
First, the stability of the system is investigated in the case of perfect sensing. A feedback control law is subsequently proposed to satisfy the design specifications. The impact of measurement noise on the followers' motion is then studied, and a control design methodology is introduced using linear matrix inequalities (LMI) to minimize the effect of noise.

\subsection{Extension Problem Formulation}

It is desired to control the followers in such a way that they follow the leader with a desired accuracy, while the leader follows an unknown trajectory. The differential equations describing the motion of the i-th robot, $\mathrm{i} \in:=\{1, \mathrm{~K}, \mathrm{n}\}$, with respect to this frame,

$$
\begin{gathered}
\dot{\mathrm{x}}^{\mathrm{i}}=\mathrm{v}^{\mathrm{i}} \cos \theta^{\mathrm{i}} \quad \dot{\mathrm{y}}^{\mathrm{i}}=\mathrm{v}^{\mathrm{i}} \sin \theta^{\mathrm{i}} \\
\theta^{\mathrm{i}}=\omega^{\mathrm{i}} \quad \dot{\mathrm{v}}^{\mathrm{i}}=\mathrm{a}^{\mathrm{i}}
\end{gathered}
$$

where the acceleration $a$ is the input variable. By assumption, robot 1 is the leader, and its dynamic equations are expressed as:

$$
\begin{gathered}
\dot{\mathrm{x}}^{1}=\mathrm{v}^{1} \cos \theta^{1} \quad \dot{\mathrm{y}}^{1}=\mathrm{v}^{1} \sin \theta^{1} \\
\theta^{1}=\omega^{1} \quad \dot{\mathrm{v}}^{1}=\mathrm{a}^{1}
\end{gathered}
$$

The error vector for the $i$-th follower, $i \in\{2, K, n\}$, is subsequently defined as:

$$
e^{i}=\left[\begin{array}{l}
e_{p}^{i} \\
e_{v}^{i}
\end{array}\right]
$$

where $e_{p}^{i}$ and $e_{v}^{i}$ are position and velocity error of the $i-t h$ follower, respectively, and are defined by:

$$
e_{p}^{i}:=\left[\begin{array}{c}
e_{p_{x}}^{i} \\
e_{p_{y}}^{i}
\end{array}\right]=\left[\begin{array}{l}
x^{i}-x^{i-1}-d_{x}^{i}(t) \\
y^{i}-y^{i-1}-d_{y}^{i}(t)
\end{array}\right]
$$

and

$e_{v}^{i}:=\left[\begin{array}{c}e_{v_{x}}^{i} \\ e_{v_{y}}^{i}\end{array}\right]=\left[\begin{array}{c}\dot{x}^{i}-\dot{x}^{i-1} \\ \dot{y}^{i}-\dot{y}^{i-1}\end{array}\right]$

where $d_{x}^{i}(t)$ and $d_{y}^{i}(t)$ represent the desired relative position between $i-t h$ and $i-1$ th robots in the platoon in the $x$ and $y$ directions, respectively. The objective here is to make the position and velocity errors as close as possible to zero. It is to be noted that if $e_{v_{x}}^{i}$ and $e_{v_{y}}^{i}$ tend to zero, then $\boldsymbol{\theta}^{i} \rightarrow \boldsymbol{\theta}^{i-1}$. This means that if the velocity error approaches zero by time, then the orientation alignment of all robots in the platoon is guaranteed in the steady state.

Assumption 1. The desired relative positions $d_{x}^{i}(t)$ and $d_{y}^{i}(t)$ are either constant or the outputs of an autonomous system represented by

$$
\dot{q}(t)=\Gamma q(t) \quad d_{x}^{i}(t)=I_{x} q(t) \quad d_{y}^{i}(t)=I_{y} q(t)
$$


where $q \in R^{k}$. Note that all the eigenvalues of the $\Gamma$ lie in the open left half-plane except the one which is located in the origin.

Denote with $-\lambda$ the rightmost non-zero eigenvalues of $\boldsymbol{\Gamma}$. It is supposed that each follower is equipped with the proper sensors to measure its relative position and velocity (with respect to its preceding robot). Thus, the error vector $e^{i}$ can be used in constructing the control input. Now, using equations (16) and (17), one can write:

$\dot{e}_{p}^{i}=\left[\begin{array}{c}\dot{e}_{p_{x}}^{i} \\ \dot{e}_{p_{y}}^{i}\end{array}\right]=\left[\begin{array}{c}e_{v_{x}}^{i}-\dot{d}_{x}^{i}(t) \\ e_{v_{y}}^{i}-\dot{d}_{y}^{i}(t)\end{array}\right]$

and similarly:

$\dot{e}_{v}^{i}=\left[\begin{array}{c}\dot{e}_{v_{x}}^{i} \\ \dot{e}_{v_{y}}^{i}\end{array}\right]=\left[\begin{array}{c}\dot{v}^{i} \cos \theta^{i}-v^{i} \theta^{i} \sin \theta^{i}-\ddot{x}^{i-1} \\ \dot{v}^{i} \sin \theta^{i}+v^{i} \theta^{i} \cos \theta^{i}-\ddot{y}^{i-1}\end{array}\right]$

By rewriting the above equations and using the relations $\theta^{\mathrm{i}}=\omega$ and $\dot{\mathrm{v}}^{\mathrm{i}}=\mathrm{a}^{\mathrm{i}}$, it can be shown that

$\dot{e}_{v}^{i}=\left[\begin{array}{c}\dot{e}_{v_{x}}^{i} \\ \dot{e}_{v_{y}}^{i}\end{array}\right]=\left[\begin{array}{cc}\cos \theta^{i} & -v^{i} \sin \theta^{i} \\ \sin \theta^{i} & v^{i} \cos \theta^{i}\end{array}\right]\left[\begin{array}{l}a^{i} \\ \theta^{i}\end{array}\right]=\left[\begin{array}{c}\ddot{x}^{i-1} \\ \ddot{y}^{i-1}\end{array}\right]$

Define:

$\left[\begin{array}{l}u_{1}^{i} \\ u_{2}^{i}\end{array}\right]=\left[\begin{array}{cc}\cos \boldsymbol{\theta}^{i} & -v^{i} \sin \boldsymbol{\theta}^{i} \\ \sin \boldsymbol{\theta}^{i} & v^{i} \cos \boldsymbol{\theta}^{i}\end{array}\right]\left[\begin{array}{l}a^{i} \\ \omega^{i}\end{array}\right]$

then:

$\left[\begin{array}{c}\dot{e}_{v_{x}}^{i} \\ \dot{e}_{v_{y}}^{i}\end{array}\right]=\left[\begin{array}{c}u_{1}^{i} \\ u_{2}^{i}\end{array}\right]-\left[\begin{array}{c}\ddot{x}^{i-1} \\ \ddot{y}^{i-1}\end{array}\right]$

Combining the two equations, the error dynamics can be expressed as:

$\left[\begin{array}{c}\dot{\mathrm{e}}_{\mathrm{p}_{\mathrm{x}}}^{\mathrm{i}} \\ \dot{\mathrm{e}}_{\mathrm{p}_{\mathrm{y}}}^{\mathrm{i}} \\ \dot{\mathrm{e}}_{\mathrm{v}_{\mathrm{x}}}^{\mathrm{i}} \\ \dot{\mathrm{e}}_{\mathrm{v}_{\mathrm{y}}}^{\mathrm{i}}\end{array}\right]=\underbrace{\left[\begin{array}{cccc}0 & 0 & 1 & 0 \\ 0 & 0 & 0 & 1 \\ 0 & 0 & 0 & 0 \\ 0 & 0 & 0 & 0\end{array}\right]}_{\mathrm{A}}\left[\begin{array}{c}\mathrm{e}_{\mathrm{p}_{\mathrm{x}}}^{\mathrm{i}} \\ \mathrm{e}_{\mathrm{p}_{\mathrm{y}}}^{\mathrm{i}} \\ \mathrm{e}_{\mathrm{v}_{\mathrm{x}}}^{\mathrm{i}} \\ \mathrm{e}_{\mathrm{v}_{\mathrm{y}}}^{\mathrm{i}}\end{array}\right]+\underbrace{\left[\begin{array}{ll}0 & 0 \\ 0 & 0 \\ 1 & 0 \\ 0 & 1\end{array}\right]}_{\mathrm{B}}\left[\begin{array}{l}\mathrm{u}_{1}^{\mathrm{i}} \\ \mathrm{u}_{2}^{\mathrm{i}}\end{array}\right]-$

$\underbrace{\left[\begin{array}{ll}0 & 0 \\ 0 & 0 \\ 1 & 0 \\ 0 & 1\end{array}\right]}_{B}\left[\begin{array}{c}\ddot{\mathrm{x}}^{\mathrm{i}-1} \\ \ddot{\mathrm{y}}^{\mathrm{i}-1}\end{array}\right]+\underbrace{\left[\begin{array}{c}\dot{-}_{\mathrm{x}}^{\mathrm{i}}(\mathrm{t}) \\ -\dot{\mathrm{d}}_{\mathrm{y}}^{\mathrm{i}}(\mathrm{t}) \\ 0 \\ 0\end{array}\right]}_{\mathrm{f}(\mathrm{t})}$

or equivalently:
$\dot{e}^{i}=A e^{i}+B u^{i}-B s^{i-1}+\phi(t)$

where $u^{i}$ denotes the control input and $s^{i-1}$ is defined as:

$S^{i-1}:=\left[\begin{array}{c}\ddot{x}^{i-1} \\ \ddot{y}^{i-1}\end{array}\right]$

Note that Assumption 1 implies $\|\phi(t)\|$ is an exponentially decaying signal.

Assumption 2. It is assumed that each robot's acceleration is uniformly bounded; i.e.,

$\left\|s^{i-1}\right\| \leq \boldsymbol{\sigma}$ for $i \in\{2, K, n\}$ where $\boldsymbol{\sigma}$ is a known constant:

A control law of the following form is proposed for the following:

$$
u^{i}=K^{i} e^{i}-\frac{B^{T} P^{i} e^{i}}{\left\|B^{T} P^{i} e^{i}\right\|}, i \in\{2, K, n\}
$$

to regulate the position and velocity errors for each follower, where $K^{i} \in R^{2 \times 4}$ is a constant matrix and $P^{i} \in R^{4 \times 4}$ is a symmetric positive definite matrix.

Remark 1. In the case when $\left\|B^{T} P^{i} e^{i}\right\|$ in (22) is "close" to zero, the control input can be modified as follows:

$$
u^{i}=\left\{\begin{array}{ll}
K^{i} e^{i}-\frac{B^{T} P^{i} e^{i}}{\left\|B^{T} P^{i} e^{i}\right\|} & \left\|B^{T} P^{i} e^{i}\right\| \geq \boldsymbol{\rho} \\
K^{i} e^{i}+\boldsymbol{\rho} & \left\|B^{T} P^{i} e^{i}\right\|<\boldsymbol{\rho}
\end{array}, i \in\{2, K, n\}\right.
$$

where $\boldsymbol{\rho}$ is a sufficiently small positive constant. It is desired to design the control law for the followers such that the steady-state error is as close to zero as possible.

\subsection{Main Results}

\subsubsection{Perfect Sensing without Input Constraint}

Theorem 4. Consider a platoon of WMRs moving in formation with leader-follower structure, where the dynamics of the followers obeys equation (21), and suppose the conditions of Assumptions 1 and 2 hold. Given $\boldsymbol{\alpha}>0$, also assume that there exist matrices $R^{i}>0$ and $S^{i}$ satisfying the following LMI:

$$
R^{i} A^{T}+A R^{i}+S^{i^{T}} B^{T}+B S^{i}+\boldsymbol{\alpha} R^{i}<0
$$

If the controller (22) with $\mathrm{K}^{\mathrm{i}}=\mathrm{S}^{\mathrm{i}} \mathrm{R}^{\mathrm{i}^{-1}}$ and $\mathrm{P}^{\mathrm{i}}=\mathrm{R}^{\mathrm{i}^{-1}}$ is applied to the follower $i$, then $\left\|e^{i}(t)\right\|$ decays exponentially.

\subsubsection{Perfect Sensing with Input Constraint}

Theorem 5. Consider the system described in Theorem 4. Define

$\boldsymbol{\mu}_{1}=\max \left\|e_{p_{x}}{ }^{i}\right\|=\max \left\|e_{p_{y}}{ }^{i}\right\|$ 
and

$\mu_{2}=\max \left\|e_{v_{x}}^{i}\right\|=\max \left\|e_{v_{y}}^{i}\right\|$,

and let the design parameters $\boldsymbol{\alpha}>0, \boldsymbol{\eta}>0$ be given. Solve the following LMIs

$$
\begin{aligned}
& R^{i} A^{T}+A R^{i}+S^{i^{T}} B^{T}+B S^{i}+\boldsymbol{\alpha} R^{i}<0 \\
& {\left[\begin{array}{cc}
\boldsymbol{\eta}^{2} R^{i} & S^{i^{T}} \\
S^{i} & I_{2}
\end{array}\right]>0} \\
& R^{i}>4\left[\begin{array}{cc}
\boldsymbol{\mu}_{1}^{2} I_{2} & 0 \\
0 & \boldsymbol{\mu}_{2}^{2} I_{2}
\end{array}\right]
\end{aligned}
$$

where $I_{2}$ is the $2 \times 2$ Identity matrix. If the problem has a feasible solution, then

1: If the controller (22) with $\mathrm{K}^{\mathrm{i}}=\mathrm{S}^{\mathrm{i}} \mathrm{R}^{\mathrm{i}^{-1}}$ and $\mathrm{P}^{\mathrm{i}}=\mathrm{R}^{\mathrm{i}^{-1}}$ is applied to the follower $i$, then $\left\|e^{i}(t)\right\|$ is an exponentially decaying signal, and 2: $\left\|\mathrm{u}^{\mathrm{i}}\right\|<\boldsymbol{\eta}+\boldsymbol{\sigma}$

\subsubsection{Noisy Measurements}

The control law (22) in the presence of measurement noise on the follower's motion can be written as:

$\mathrm{u}^{\mathrm{i}}=\mathrm{K}^{\mathrm{i}} \tilde{\mathrm{e}}^{\mathrm{i}}-\frac{\mathrm{B}^{\mathrm{T}} \mathrm{P}^{\mathrm{i}} \tilde{\mathrm{e}}^{\mathrm{i}}}{\left\|\mathrm{B}^{\mathrm{T}} \mathrm{P}^{\mathrm{i}} \tilde{\mathrm{e}}^{\mathrm{i}}\right\|} \boldsymbol{\sigma}$

where $\tilde{\mathrm{e}}^{\mathrm{i}}=\mathrm{e}^{\mathrm{i}}+\delta_{\mathrm{e}}^{\mathrm{i}}$, and $\boldsymbol{\delta}_{\mathrm{e}}^{\mathrm{i}}$ is the measurement noise, which is assumed to have a known bound represented by:

$$
\Delta_{\mathrm{e}}^{\mathrm{i}}:=\underbrace{\max }_{\mathrm{t}>\mathrm{t}_{0}}\left\|\delta_{\mathrm{e}}^{\mathrm{i}}\right\|^{2}
$$

In this subsection, an upper bound on the steady-state error is obtained and an algorithm is proposed to design $\mathrm{K}^{\mathrm{i}}$ and $\mathrm{P}^{i}>0$ such that this upper bound is minimized. To this end, the following lemma is presented,

Lemma 2. Assume that $\mathrm{g}(\mathrm{t})$ is an exponentially decaying signal. Given $\xi>0,0$, let the following inequality hold:

$$
\dot{\mathrm{V}}+\xi \mathrm{V}-\mathrm{b} \delta_{\mathrm{e}}^{\mathrm{iT}} \mathrm{Q} \delta_{\mathrm{e}}^{\mathrm{i}}-\|\mathrm{g}(\mathrm{t})\|^{2}<0
$$

where $\mathrm{b}$ is a positive constant and $\mathrm{Q}$ is a symmetric positive definite matrix. Then:

$$
\mathrm{V}(\infty)<\frac{\mathrm{b}}{\xi} \underbrace{\max }_{\mathrm{t}>\mathrm{t}_{0}}\left[\delta_{\mathrm{e}}^{\mathrm{iT}}(\mathrm{t}) \mathrm{Q} \delta_{\mathrm{e}}^{\mathrm{i}}(\mathrm{t})\right]
$$

Remark 2. Consider the system described in Theorem 3.1. Let $R^{i}=P^{i^{-1}} i$ and assume $R^{i}$ has a lower bound $R_{1}$ and an upper bound $R_{r}$ given in (25) and (26), respectively. Using the result of Theorem 2.3 for each follower, it can be concluded that, if the controller (27) with $K^{i}=S^{i} R^{i^{-1}}$ and $\mathrm{P}^{\mathrm{i}}=\mathrm{R}^{\mathrm{i}^{-1}}$ is applied to the follower $\mathrm{i}$, then:

1: $\left\|e^{i}(t)\right\|$ exponentially decaying signal for $\Delta_{e}^{i}=0$.

2: $\lim _{t \rightarrow \infty}\left\|e_{p}^{i}\right\|<\bar{e}_{p, \min }$

An upper bound on the steady-state position error is obtained as $\left\|\mathrm{e}^{\mathrm{p}}(\infty)\right\|^{2}$ and Remark 1 holds. This is can be proved by choosing $Q=R^{i^{-1}}$ and $\boldsymbol{\gamma}$ selected as $\gamma<(\alpha-\xi) \lambda_{\min }\left(\mathrm{P}^{\mathrm{i}}\right)$.

$g(t)=\frac{1}{\sqrt{\gamma}} P^{i} f(t)$

\section{SIMULATION RESULT (SINGLE ROBOT)}

In case 1 the results obtained for the tracking problem of a single robot in the presence of input constraint are examined by simulations.

\subsection{Case 1}

Consider a WMR, and let the desired trajectory to be followed by the robot be a path for circular trajectory given by

$$
x_{r}=2 \cos 0.025 t, \quad y_{r}=2 \sin 0.025 t
$$

It is desired to obtain a control input of the form (9) in which the magnitude of a is less than $\boldsymbol{\eta}$ at all times, while the tracking objective described above is achieved.

Assume that the experiment is to be performed in a $4 \times 4$ $\mathrm{m}$ environment; hence,

$$
\max \left\|e_{p_{x}}\right\|=\max \left\|e_{p_{y}}\right\|=\boldsymbol{\mu}_{1}=4
$$

Furthermore, let the maximum speed of the robot be 0.3 $\mathrm{m} / \mathrm{sec}$, i.e.,

$\max \left\|e_{\mathrm{V}_{X}}\right\|=\max \left\|e_{\mathrm{V}_{Y}}\right\|=\boldsymbol{\mu}_{2}=0.3$

Let also $\boldsymbol{\eta}=0.04$; one can then use Theorem 2 with $\boldsymbol{\alpha}=0.04$ and $\varepsilon_{0}=2 \times 10^{-9}$ to obtain the gain matrix $\mathrm{K}$ in (9) as:

$K=\left[\begin{array}{cccc}-0.0007 & 0 & -0.0469 & 0 \\ 0 & -0.0007 & 0 & -0.0469\end{array}\right]$

Let $\alpha=0.04$ and $b=1.55$ Assume that the measurement noise is a random process, which is uniformly distributed in the intervals $\left(0,2 \times 10^{-5}\right)$ and $\left(0,10^{-3}\right)$ for position and velocity measurements, respectively. Fig. (4) shows that the velocity regulation error $\mathrm{e}_{\mathrm{v}}$ approaches zero in both $\mathrm{x}$ and $\mathrm{y}$ directions. Fig. (5) shows the trajectory of the robot moving toward the circular path from its initial position $(1.9,0.1)$. 


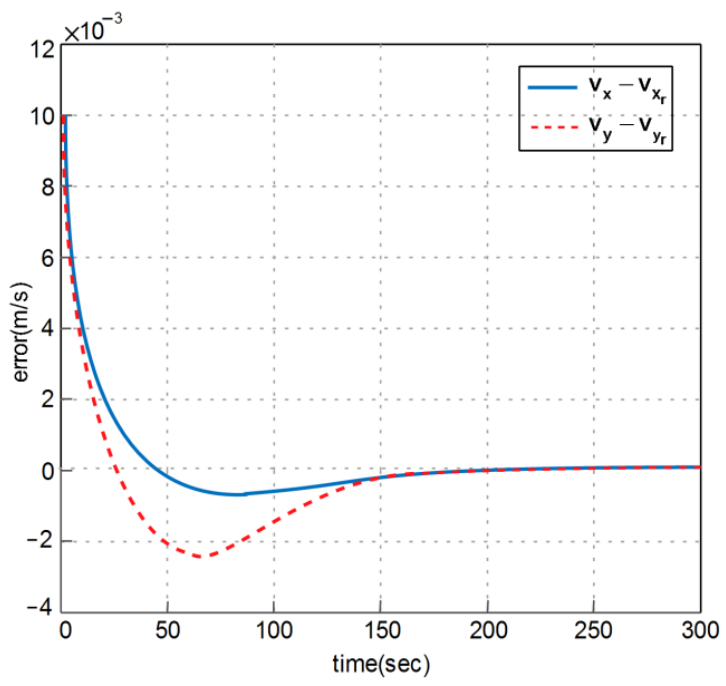

Fig. (4). The velocity error of the robot.

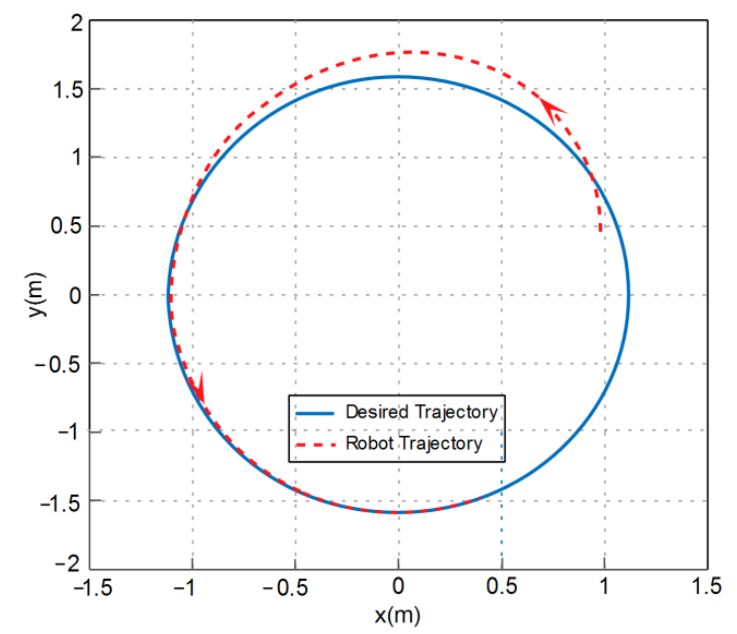

Fig. (5). The robot's trajectory in the 2-D plane.

The norm of the control input for the robot is depicted in Fig. (6), which shows that the input constraint is fulfilled. The planar motion of the formation is sketched in Fig. (7).

\section{SIMULATION RESULTS (FORMATION)}

\subsection{Case 1}

Consider two mobile robots, one leader and one follower, and assume the leader moves on a circular track given by:

$\mathrm{x}_{\mathrm{r}}^{1}=2 \cos 0.025 \mathrm{t}, \mathrm{y}_{\mathrm{r}}^{1}=2 \sin 0.025 \mathrm{t}$

The follower is to follow the leader with the following desired distance:

$d(t)=\left[\begin{array}{l}d_{x}(t) \\ d_{y}(t)\end{array}\right]=\left[1-2\left(1-e^{-t}\right)\right]\left[\begin{array}{c}1 \\ -1\end{array}\right]$
In this case, the gain matrix $K$ in (3.8) will be:

$\mathrm{K}=\left[\begin{array}{cccc}-0.0007 & 0 & -0.0420 & 0 \\ 0 & -0.0007 & 0 & -0.0420\end{array}\right]$

In Fig. (8). the relative position of the follower with respect to the leader along the $\mathrm{x}$-axis is compared with its desired trajectory $\mathrm{d}_{\mathrm{x}}$.

A similar comparison is made in the $y$ direction in Fig. (9). These figures demonstrate that the desired position tracking is achieved asymptotically.

Furthermore, Fig. (10) shows that the velocity regulation error $e_{v}$ approaches zero in both directions $x$ and $y$. Fig. (11) shows the trajectory of the leader and follower moving toward the circular path from their initial positions $(1.9,0.1)$ 


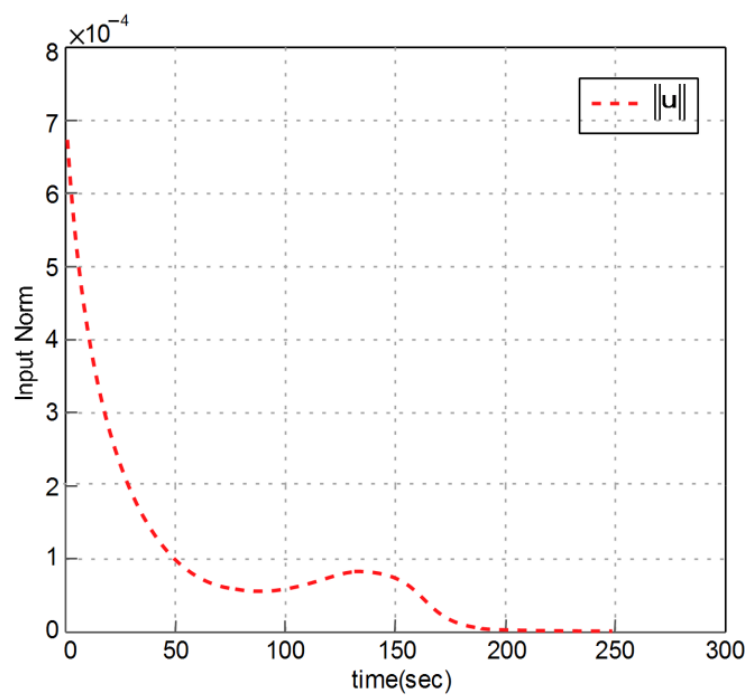

Fig. (6). The norm of the control input $\|u\|$.

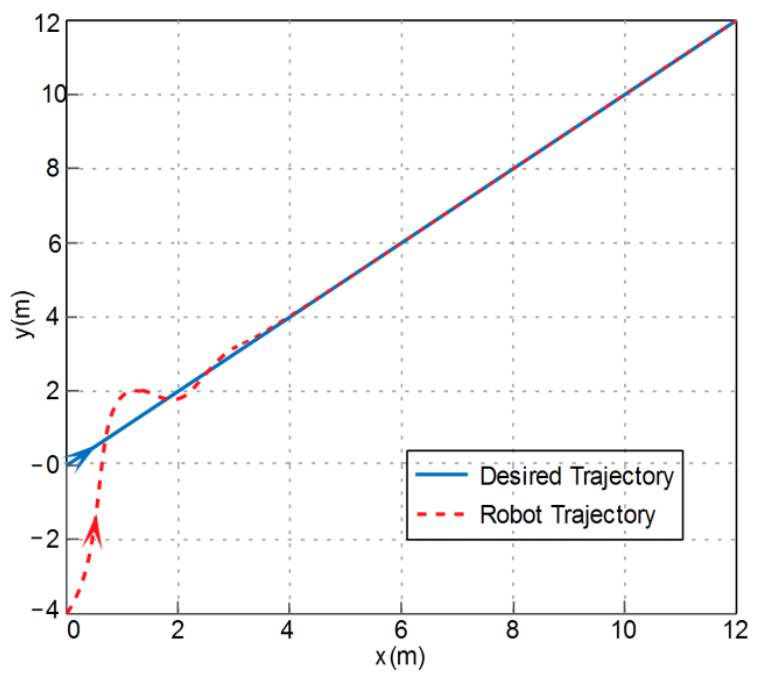

Fig. (7). The robot's trajectory in the 2-D plane for the linear trajectory.

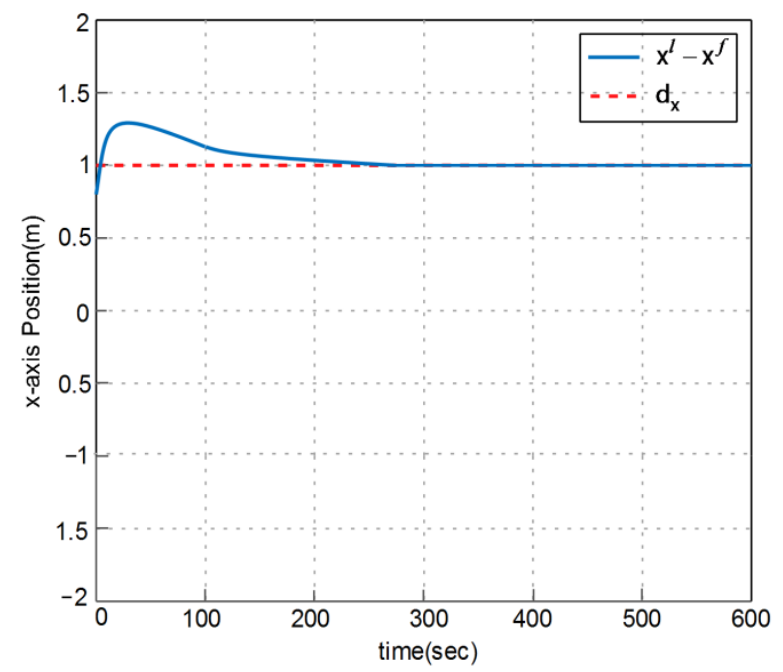

Fig. (8). Relative position of the follower with respect to the leader along the x-axis. 


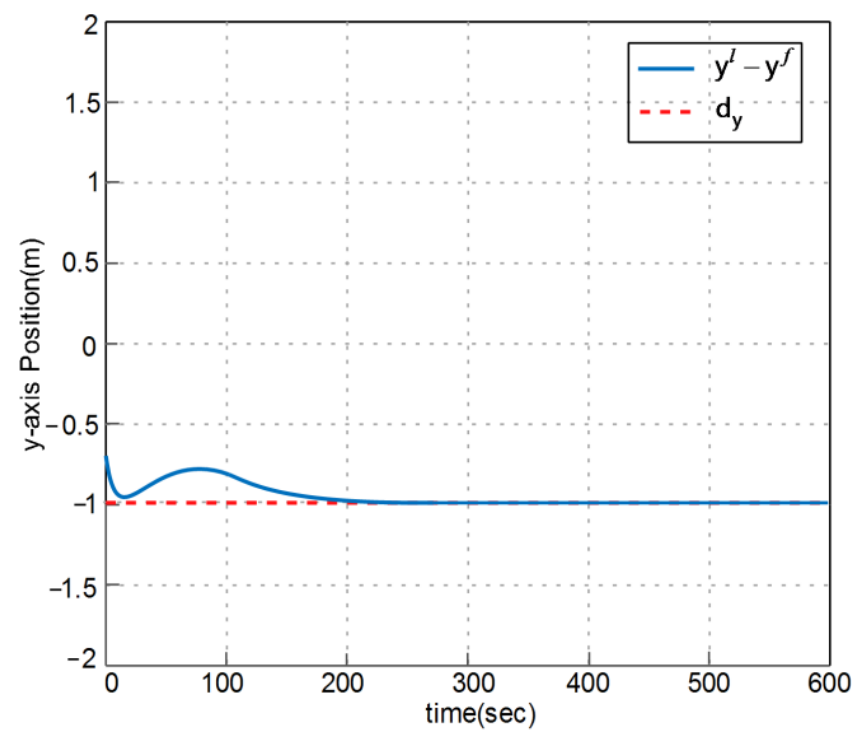

Fig. (9). Relative position of the follower with respect to the leader along the y-axis.

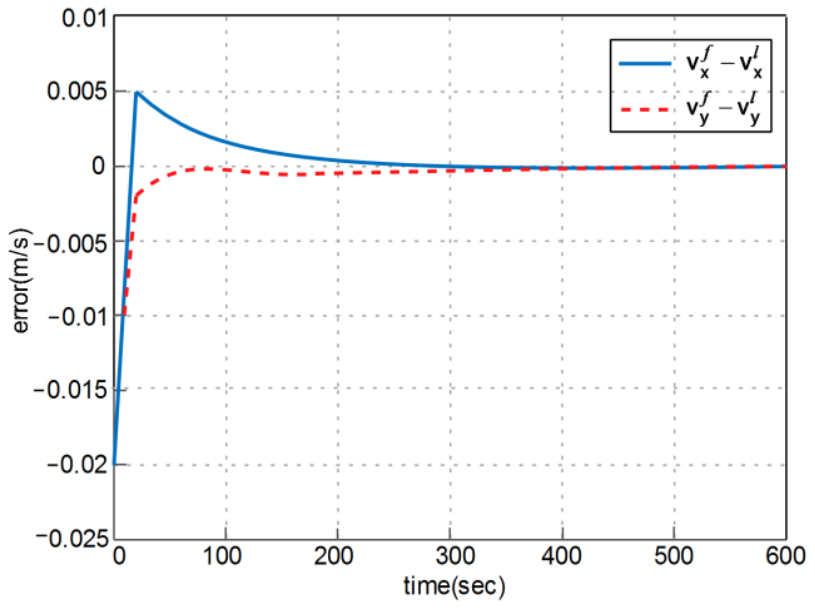

Fig. (10). The velocity error of the follower for the leader-follower trajectory tracking.

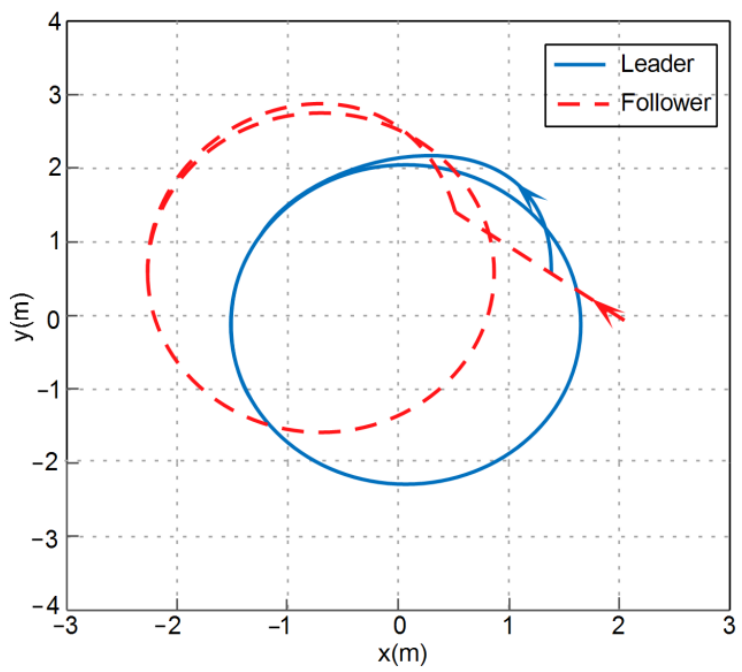

Fig. (11). The leader and follower trajectories in the 2-D plane for the leader-follower trajectory tracking. 


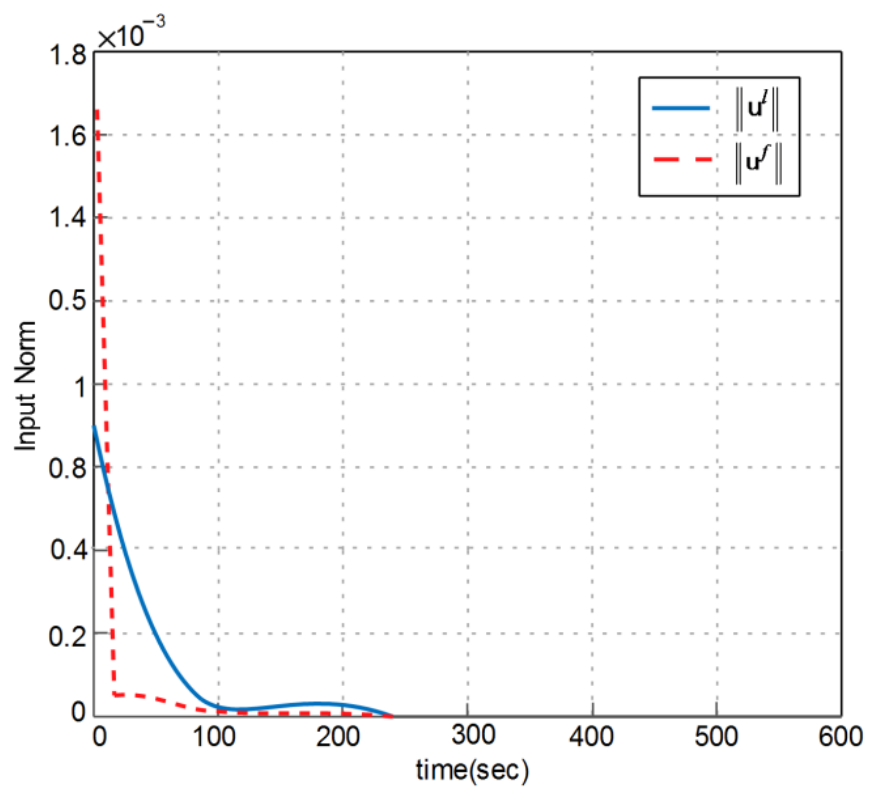

Fig. (12). Control input norms $\left\|u^{1}\right\|$ and $\left\|u^{\mathrm{f}}\right\|$ for the leader-follower trajectory tracking.

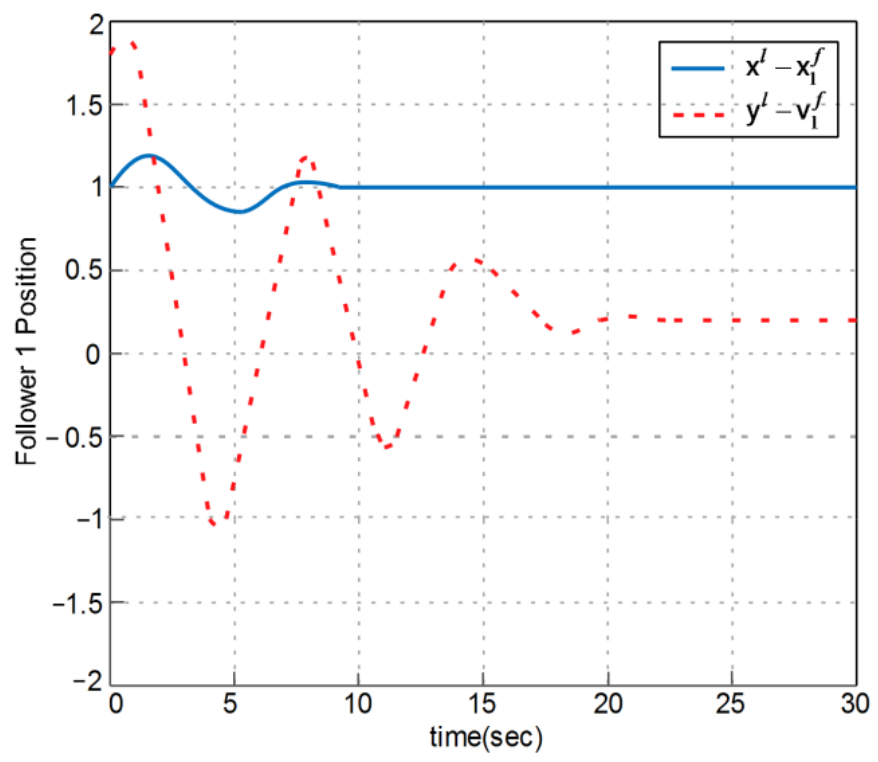

Fig. (13). Relative position of follower 1 with respect to the leader along the $x$ and $y$ axis.

and $(2.8,-0.8)$, respectively. The norm of the control inputs applied to the follower and leader, $\left\|u^{1}\right\|$ and $\left\|u^{f}\right\|$ is depicted in Fig. (12), which demonstrates that the input constraint is satisfied.

\subsection{Case 2}

Consider a multi-agent system, where 2 followers are to follow a leader in a linear path. Suppose that the leader and followers are initially located on an equilateral triangle with the length of the sides equal to $2 \mathrm{~m}$. The final desired formation is another equilateral triangle with the length of the sides equal to $1 \mathrm{~m}$, while the leader is tracking a ramp reference signal along both axes, characterized by: $x_{r}^{l}(t)=y_{r}^{l}(t)=0.4 t$

Let $\alpha=0.04$ and $b=1.55$. Assume that the measurement noise is a random process which is uniformly distributed in the intervals $\left(0,2 \times 10^{-5}\right)$ and $\left(0,10^{-3}\right)$ for position and velocity measurements, respectively. The gain matrix given below is obtained for both followers:

$$
\mathrm{K}=\left[\begin{array}{cccc}
-1.6724 & 0 & -0.7615 & 0 \\
0 & -1.6724 & 0 & -0.7615
\end{array}\right]
$$

Fig. (13) depicts the relative position of follower 1 with respect to the leader in both directions. The velocity regulation error of follower 1 along the $\mathrm{x}$ and $\mathrm{y}$ axes is plotted in 


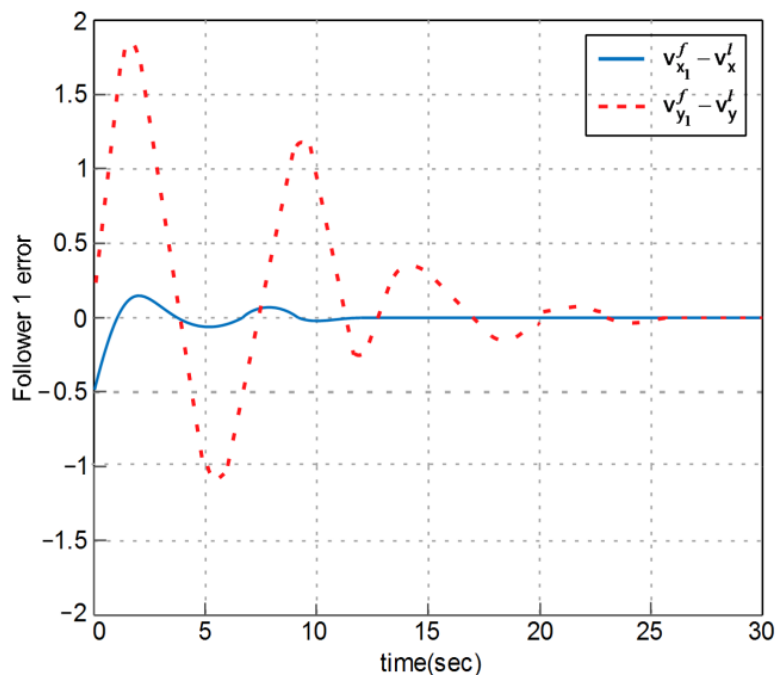

Fig. (14). The velocity of follower 1 for the leader-follower trajectory tracking.

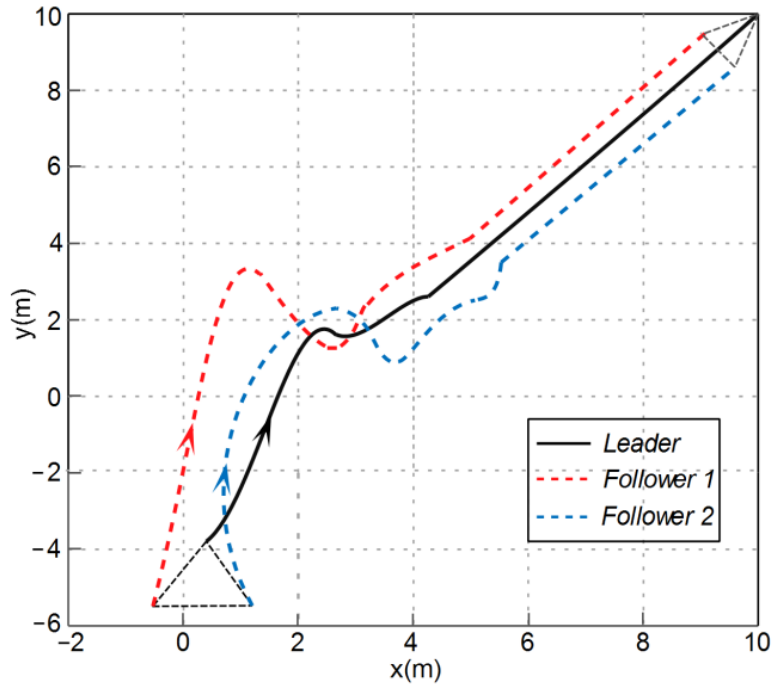

Fig. (15). The planar motion of the formation for the leader-follower trajectory tracking.

Fig. (14). This figure shows that the error approaches zero in both directions. The planar motion of the formation is sketched (Fig. 15).

\section{CONCLUSION}

Mobile robots are the focus of a great deal of current research in robotics. The objectives of this paper are to derive a control law for a single wheeled mobile robot, so that system remains stable while tracking a trajectory and to design a controller for the leader-follower formation problem so that the system is stable in the cases of perfect sensing and perfect sensing with input constraint. Upper bounds for the steady-state position and velocity errors are obtained in the presence of measurement noise. Using linear matrix inequalities (LMI), a proper controller is designed to stabilize the system under different conditions. Input saturation is also addressed by imposing a proper constraint on robot's input. Moreover, upper bounds for the steady-state position and velocity errors are found. Formation control for a group of mobile unicycle robots is then studied, input saturation is also addressed by imposing a proper constraint on the followers' input in the formulation. Stability analysis is provided, and a controller is designed using LMIs to minimize the upper bound of the steady-state errors. The drawback in the present work is that an obstacle free environment is considered. However, in real environments, there often exist obstacles which need to be considered in designing the controller. It is assumed that each robot's acceleration bound is known. However, this is not a practical assumption in some applications. One can use an adaptive control scheme to relax this condition. Examples of path following are examined by simulation, which demonstrate the efficacy of the proposed methods. 


\section{CONFLICT OF INTEREST}

The author confirms that this article content has no conflict of interest.

\section{ACKNOWLEDGEMENTS}

Declared none.

\section{REFERENCES}

[1] Y. Kanayama, Y. Kimura, F. Miyazaki, and T. Noguchi, "A stable tracking control method for an autonomous mobile robot", in Proceedings of the IEEE International Conference on Robotics and Automation, May 1990, vol. 1, pp. 384-389.

[2] W. Dong, "Tracking control of multiple-wheeled mobile robots with limited information of a desired trajectory", IEEE Transactions on Robotics, vol. 28, pp. 262-268, 2012.

[3] Nicoleta Minoiu Enache, "Trajectory Tracking Control by LMIbased Approach for Car-like Robots Nicoleta Minoiu Enache", In Proceedings of the 9th International Conference on Informatics in Control, Automation and Robotics, 2012, Rome, Italy, pp. 38-47.

[4] C. Samson and K. Ait-Abderrahim, "Feedback control of a nonholonomic wheeled cart in Cartesian space", in Proceedings of the IEEE International Conference on Robotics and Automation, 1991, vol. 2, pp. 1136-1141.

[5] Z. L. Liu, "Evaluation on developing level of unban agglomeration derived from resources exploration", Journal of Applied Sciences, vol. 13, no. 21, pp. 4702-4707, 2013.

[6] B. d'Andrea Novel, G. Campion, and G. Bastin, "Control of nonholonomic wheeled mobile robots by state feedback linearization", The International Journal of Robotics Research, vol. 14, no. 6, pp. 543-559, 1995.

[7] Z. P. Jiang and H. Nijmeijer, "A recursive technique for tracking control of nonholonomic systems in chained form”, IEEE Transactions on Automatic Control, vol. 44, No.2, pp. 265-279, 1999.

[8] R. Fierro and F. Lewis, "Control of a nonholonomic mobile robot: backstepping kinematics into dynamics", in Proceedings of the 34th IEEE Conference on Decision and Control, 1995, vol. 4, pp. 3805-3810.
[9] H. Durrant-Whyte and T. Bailey, "Simultaneous localization and mapping: part I", IEEE Robotics \& Automation Magazine, vol. 13, no. 2, pp. 99-110, 2006.

[10] T. van den Broek, N. van de Wouw, and H. Nijmeijer, "Formation control of unicycle mobile robots: a virtual structure approach", in Proceedings of Joint 48fft IEEE Conference on Decision and Control and 2\&th Chinese Control Conference, 2009, pp. 8328-8333.

[11] J. Chen, D. Sun, J. Yang, and H. Chen, "Leader-follower formation control of multiple non-holonomic mobile robots incorporating a receding-horizon scheme", The International Journal of Robotics Research, vol. 29, no. 6, pp. 727-747, 2010.

[12] Z. Peng, G. Wen, A. Rahmani, and Y. Yu, "Distributed consensus-based formation control for multiple nonholonomic mobile robots with a specified reference trajectory", International Journal of Systems Science, 2013.

[13] I. Zohar, A. Ailon, and R. Rabinovici, "Mobile robot characterized by dynamic and kinematic equations and actuator dynamics: trajectory tracking and related application", Robotics and Autonomous Systems, vol. 59, no. 6, pp. 343-353, 2011.

[14] R. Fierro and F. L. Lewis, "Control of a nonholonomic mobile robot using neural networks", IEEE Transactions on Neural Networks, vol. 9, no. 4, pp. 589-600, 1998

[15] T. Fukao, H. Nakagawa, and N. Adachi, "Adaptive tracking control of a nonholonomic mobile robot", IEEE Transactions on Robotics and Automation, vol. 16, No. 5, pp. 609-615, 2000.

[16] Z. Wang, Y. Takano, Y. Hirata, and K. Kosuge, "Decentralized cooperative object transportation by multiple mobile robots with a pushing leader", Distributed Autonomous Robotic Systems, pp. 453-462, 2007.

[17] R. Bishop, Intelligent Vehicle Technology and Trends. Artech House, 2005.

[18] W. Burgard, M. Moors, C. Stachniss, and F. Schneider, "Coordinated multi-robot exploration", IEEE Transactions on Robotics, vol. 21, no. 3, pp. 376-386, 2005.

[19] P. Corke, J. Trevelyan, T. Sugar, and V. Kumar, "Control and coordination of multiple mobile robots in manipulation and material handling tasks", Experimental Robotics VI Lecture Notes in Control and Information Sciences, vol. 250, pp. 15-24, 2000.

H. Lim, Y. Kang, C. Kim, J. Kim, and B. J. You, "Nonlinear model predictive controller design with obstacle avoidance for a mobile robot", in Mechtronic and Embedded Systems and Applications IEEE/ASME International Conference, 2008, pp. 494-499

(C) Manzoor et al.; Licensee Bentham Open.

This is an open access article licensed under the terms of the Creative Commons Attribution-Non-Commercial 4.0 International Public License (CC BY-NC 4.0) (https://creativecommons.org/licenses/by-nc/4.0/legalcode), which permits unrestricted, non-commercial use, distribution and reproduction in any medium, provided the work is properly cited. 